\title{
Ets-1 is implicated in the regulation of androgen co-regulator FHL2 and reveals specificity for migration, but not invasion, of $\mathrm{PC} 3$ prostate cancer cells
}

\author{
DAVID ADLER $^{1}$, BERIT LANGER ${ }^{1}$, ANDREAS LINDSTROT ${ }^{1}$, ALEXANDRA FLORIN ${ }^{1}$, \\ ANJA BOSSERHOFF $^{2}$, REINHARD BUETTNER ${ }^{1}$ and NICOLAS WERNERT ${ }^{1}$ \\ ${ }^{1}$ Institute of Pathology, University Hospital Bonn, 53127 Bonn; \\ ${ }^{2}$ Institute of Pathology, University of Regensburg, 93053 Regensburg, Germany
}

Received September 26, 2010; Accepted November 1, 2010

DOI: $10.3892 /$ or.2011.1156

\begin{abstract}
Different members of the Ets-family of transcription factors are involved in TMPRSS-2-Ets translocations frequently found in human prostate cancers. We previously reported that Ets-1, which is the prototype of Ets-family members, promotes both migration and invasion of melanoma, HeLa and glioma cells. Here, we examined whether Ets-1 has a similar effect upon migration and invasion of PC3 prostate cancer cells, and whether it is implicated in the regulation of the androgen co-regulator four and a half LIM only protein-2 (FHL2). Two stable PC3 cell cultures were established by transfection with either an Ets-1 inverse antisense expression vector or a mock control vector. Western blot analysis confirmed presence of Ets-1 in mock and absence in Ets-1 inverse cells. Microarray and qRT-PCR revealed an up-regulation of FHL2 in Ets-1 blocked cells, compared to mock. To examine the effects of Ets- 1 upon cell migration, a wound assay was performed, and demonstrated that wounds were completely colonized by mock compared to Ets-1 blocked cells after 55 h. Evaluation of the effect upon invasion was examined using the Boyden chamber, which revealed no significant difference between mock and Ets-1 blocked cells. In conclusion, our study demonstrated for the first time that Ets-1 is implicated in the regulation of the androgen co-regulator FHL2, and reveals specificity of action for migration, but not invasion of PC3 prostate cancer cells.
\end{abstract}

\section{Introduction}

According to current models, tumour development and progression is a result of changes in gene expression patterns involving pathways responsible for different steps of the

Correspondence to: Dr Nicolas Wernert, Institute of Pathology, University Hospital Bonn, 53127 Bonn, Germany

E-mail: nicolas.wernert@ukb.uni-bonn.de

Key words: PC3 prostate cancer cells, migration, invasion, Ets-1 so-called metastatic cascade (such as proliferation, migration, invasion and tumour angiogenesis $(1,2)$. Particular genes involved in these pathways are classified as oncogenes, tumour suppressor genes, apoptosis-relevant genes or genes involved in DNA-repair according to their functions (2-5). The expression of these genes is tightly regulated through different combinations of transcription factors (in cooperation with co-activators and co-suppressors), which play key roles in activating or repressing transcription by binding to regulatory regions in their target genes (6).

The ETS family of transcription factors is characterized by an evolutionary highly conserved DNA-binding domain, the Ets domain, which consists of 80 amino acids with 4 tryptophane repeats (7). ETS family members have been shown to play important roles in different cell types (such as B cells, endothelial cells and fibroblasts as well as in neoplastic cells) for processes including development, differentiation, proliferation, apoptosis, migration, tissue remodelling, invasion and angiogenesis (8-12).

In prostate cancer unique chromosomal rearrangements have been found that fuse the TMPRSS2 gene, an androgenregulated prostate-specific serine protease, with several members of the ETS family, most commonly ERG (12-14). Such fusions increase expression of rearranged Ets-factors as a response to androgens, leading to an androgen-stimulated early stage prostate cancer progression (12).

Among the ETS family members, Ets-1 is the prototype that has been studied most extensively, and found to be expressed in different cell types. It has been shown to play a role in tumour progression by acting upon both stromal and neoplastic cells. Ets-1 participates in tumour angiogenesis and tumour invasion by inducing matrix-degrading proteases in both endothelial cells and fibroblasts of the tumour stroma (15-21). In neoplastic cells such as melanoma, HeLa and glioma cells, Ets-1 promotes proliferation, migration and invasion $(10,11,22)$. Ets-1 has been also found to be expressed in prostate cancer cells.

As a continuation of our efforts into understanding the implications of Ets-1 expression in neoplastic cells, we examined in this study the effects of a blockade of Ets-1 upon migration and invasion of PC 3 prostate cancer cells. We used an Ets-1 antisense vector for blockade. Further- 
more, as prostate cancer progression involves the overactivation of the androgen receptor (AR) and several of its co-activators (23-26), we decided to investigate whether Ets-1 is implicated in the regulation of the androgen co-regulator four and a half LIM only protein-2 (FHL2) in PC3 prostate cancer cells. FHL2 enhances the activity of the AR and has been found in different tissues including prostate glands (27). In prostate cancer, FHL2 has been shown to be overexpressed and correlated with poor prognosis $(25,26,28)$.

Here, we demonstrate for the first time that Ets- 1 is implicated in the regulation of the androgen co-regulator FHL2, and reveals specificity of action for migration, but not invasion of PC3 prostate cancer cells.

\section{Materials and methods}

Cell culture. PC3 cells were grown in F12K media with L-Glutamine (Invitrogen, USA) supplemented with penicillin, streptomycin (Invitrogen) and 10\% heat-inactivated fetal calf serum (Invitrogen). Into media of transfected cells, $4.3 \mathrm{mg} / \mathrm{ml}$ G418 (PAA, Austria), was added.

Cytoplasmic and nuclear extracts. Cytoplasmic and nuclear extracts were prepared from $5 \times 10^{6}$ cells as described (29). Briefly, the pelleted cells were resuspended in $300 \mu \mathrm{l}$ of buffer A containing $10 \mathrm{mM}$ Hepes, pH 7.5, $10 \mathrm{mM} \mathrm{KCl}$, $3 \mathrm{mM} \mathrm{NaCl}, 3 \mathrm{mM} \mathrm{MgCl}_{2}, 1 \mathrm{mM}$ EDTA, $1 \mathrm{mM}$ EGTA, $2 \mathrm{mM}$ dithiothreitol, protease and phosphatase inhibitors. The resuspended cells in buffer A were then incubated on ice for $15 \mathrm{~min}$. Following incubation, 0.05 volumes of $10 \%$ Nonidet P-40 were added, and cells were vortexed for $10 \mathrm{sec}$. The sample was then centrifuged at $500 \times \mathrm{g}$ for $10 \mathrm{~min}$ at $4{ }^{\circ} \mathrm{C}$. The supernatants were collected and labeled as cytoplasmic extract. The remaining pellet was washed in $200 \mu 1$ of buffer A and centrifuged as described above. The pelleted nuclei was then resuspended in $50 \mu \mathrm{l}$ of buffer B containing $20 \mathrm{mM}$ Hepes, pH 7.5, 25\% glycerol, $0.8 \mathrm{M} \mathrm{KCl}$, $1 \mathrm{mM} \mathrm{MgCl}_{2}, 1 \%$ Nonidet P-40, $0.5 \mathrm{mM}$ EDTA, $2 \mathrm{mM}$ dithiothreitol, protease and phosphatase inhibitors. The pelleted nuclei in buffer $\mathrm{B}$ were incubated on ice for $20 \mathrm{~min}$ and centrifuged at $14000 \mathrm{x}$ for $15 \mathrm{~min}$ at $4^{\circ} \mathrm{C}$. The supernatant recovered thereafter was labeled as nuclear extract. Protein concentration was then determined using ND1000 Nanodrop (Peqlab, USA). The presence of Ets-1 in the cytoplasmic and nuclear fractions was determined by Western blot analysis using anti-Ets-1 mouse polyclonal antibodies (Transduction Laboratories).

Ets-1 blockade. PC3 cells were cultured in F12K media (Invitrogen) supplemented with antibiotics and 10\% heatinactivated fetal calf serum. Plasmids pcDNA3.1h-ets-1 inverse and pcDNA3.1 (-) (mock control), respectively, were transfected into cells by the calcium phosphate method as described (30). At $24 \mathrm{~h}$ after transfection, selection was started using $4.3 \mathrm{mg} / \mathrm{ml} \mathrm{G} 418$ (PAA). The cells were then lysed by freeze and thaw at room temperature. A total of $20 \mu \mathrm{g}$ of protein was analyzed by $10 \%$ SDS-polyacrylamide gel electrophoresis and transferred onto nitrocellulose filters (Bio-Rad). The filters were then blocked with 5\% dry milk in TBST (50 mM Tris, 150 mM NaCl, 0.1\% Tween-20, pH 7.5) for $2 \mathrm{~h}$ at room temperature. The filters were then incubated in TBST and with anti-Ets-1 mouse polyclonal antibodies (1:5000; Transduction Laboratories), with anti-mouse IgG horseradish peroxidase-conjugated secondary antibody (1:5000; Amersham Biosciences). Antibodies were detected using ECL reagents (Amersham Biosciences). The housekeeping protein $B$-actin was used as an internal control and was detected using mouse monoclonal anti- $\beta$-actin antibody (1:5000; Sigma).

Wound assay. The wound-induced migration assay was performed as described (31). Briefly, PC3 Ets-1 inverse and mock control cells were suspended in F12K media with $10 \%$ FCS. Cells/well $\left(2 \times 10^{5}\right)$ were plated onto 6-well plates and incubated for $24 \mathrm{~h}$. The monolayers of the cells were then scraped with a plastic pipette tip and washed with PBS, and the medium was then replaced with F12K media with $10 \%$ FCS. The cells were photographed at 0, 24, 48 and $55 \mathrm{~h}$ after scraping.

Invasion assay. Boyden chamber invasion assay was performed as described (32) using 24-well Matrigel invasion chambers with $8-\mu \mathrm{m}$ polycarbonate filters coated with Matrigel (BD Bioscience, Bedford, MA). A fixed number of PC3 inverse or mock cells $\left(1 \times 10^{5}\right.$ cells) in routine medium containing $2 \%$ FCS were placed in the Matrigel pre-coated upper chamber inserts (BD Bioscience). The lower chamber contained routine medium with $10 \%$ FCS. Cells were incubated for $24 \mathrm{~h}$, and then the non-invading cells on the upper surface of the filter, were removed with cotton swab. The membranes were removed from the insert with a scalpel, and stained with hematoxylin (Fluka, Germany). The invading cells were counted in 4 different random fields. T-test was performed to determine the statistical significance of the data.

Microarray analysis of mRNA expression of PC3-cells. RNA was isolated from cells with the RNeasy Mini Kit (Qiagen, Germany) as described by the manufacturer. A total RNA of 38 and $17 \mu \mathrm{g}$ was recovered from PC3 Ets-1 invers cells and mock control cells, respectively. The recommended DNase digestion was carried out with the RNase-Free DNase Set (Qiagen). The amount of the isolated RNA was measured with the ND1000 Nanodrop (Peqlab). The RNA was sent to Miltenyi Biotec, Germany, for Microarray analysis and bio-informatical interpretation. The RNA was labeled with $\mathrm{Cy} 3$ and hybridized on the Whole Human Genome Oligo Microarray 4x44K (Agilent, USA) as described by the manufacturer.

Quantitative RT-PCR. The reverse transcription was performed on RNA using SuperScript III First-Strand Synthesis SuperMix for qRT-PCR (Invitrogen) as described by the manufacturer. In the real-time PCR SYBR-Green ER qPCR SuperMix (Invitrogen) reaction, a volume of $10 \mu 1$ was used. The oligonucleotides and their sequences were as follows: the housekeeping gene rPL13a: (forward), TACGCTGTGA AGGCATCAAC, rPL13a (reverse), CACCATCCGCTTTT TCTTGT. FHL2, (forward), CGAGTAAGGCACACCCA AAT, FHL2: (reverse), TCTGAATAACTTTATTGCAAG TCACA (each sample was tested in triplicate). The PCR 


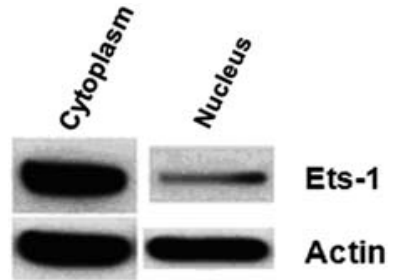

Figure 1. Western blot analysis showing Ets-1 expression in PC3 cells. Cytoplasmic and nuclear proteins from PC3 prostate cancer cells were separated on a $10 \%$ SDS-PAGE, transferred to PVDF membranes and blotted with Ets-1 specific antibodies.

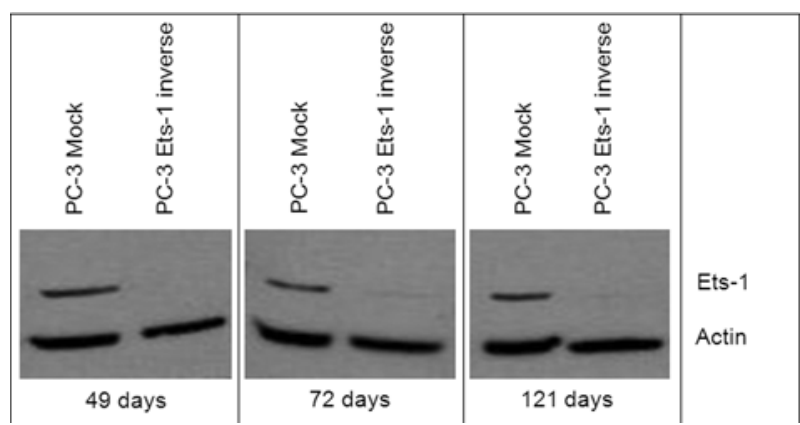

Figure 2. Stable transfection of Ets-1 in PC3 cells. Western blot analysis showing stable transfection over a period of 121 days of PC3 cells with empty plasmid (Mock) and plasmid carrying Ets-1 inverse.

conditions in the Applied Biosystems 7900HT instrument were as follows: initially $2 \min 50^{\circ} \mathrm{C}, 10 \mathrm{~min} 95^{\circ} \mathrm{C}, 40$ cycles $15 \sec 95^{\circ} \mathrm{C}$ and $60^{\circ} \mathrm{C}$ for $1 \mathrm{~min}$. Thereafter, a melting point analysis was performed with $15 \sec 95^{\circ} \mathrm{C}, 15 \sec 60^{\circ} \mathrm{C}$ and $15 \sec 95^{\circ} \mathrm{C}$. The data were collected after the $60^{\circ} \mathrm{C}$ step at every cycle and after the melting point analysis.

Data processing. Absolute Ct-values were assessed with SDS 2.2 Software (Applied Biosystems) and transferred to Microsoft Excel for further analysis. The Ct-Values were normalized with the reference to the rPL13a as the housekeeping gene. After that the $2^{-\Delta \Delta C t}$ was calculated with the values of the mock control in cell culture as a second reference. SEM is shown in the error bar.

\section{Results}

Ets-1 is expressed in PC3 prostate cancer cells and can be experimentally blocked. Ets-1 is expressed in both the cytoplasm and the nucleus in PC3 prostate cancer cells as shown by Western blot analysis (Fig. 1). Therefore, two stable PC3 cell cultures were established by transfection with either an Ets-1 invers antisense expression vector or a mock control vector. Using Western blot analysis, we found that PC3 cells expressing the mock control showed detectable amounts of Ets-1, whereas PC3 cells expressing the Ets-1 invers lacked detectable amounts of Ets-1 over a period of 121 days (Fig. 2).

Up-regulation of FHL2 in Ets-1 blocked PC3 prostate cancer cells. Based on our microarray data analysis, we observed
Table I. Microarray analysis of FHL2 expression in PC3 cells.

\begin{tabular}{lc}
\hline & Fold change \\
\hline PC3 Mock & 0.00 \\
PC3 Ets-1 inverse & 2.36 \\
\hline
\end{tabular}

The values are the result of the ratio PC3 Ets-1 inverse to PC3 Mock.

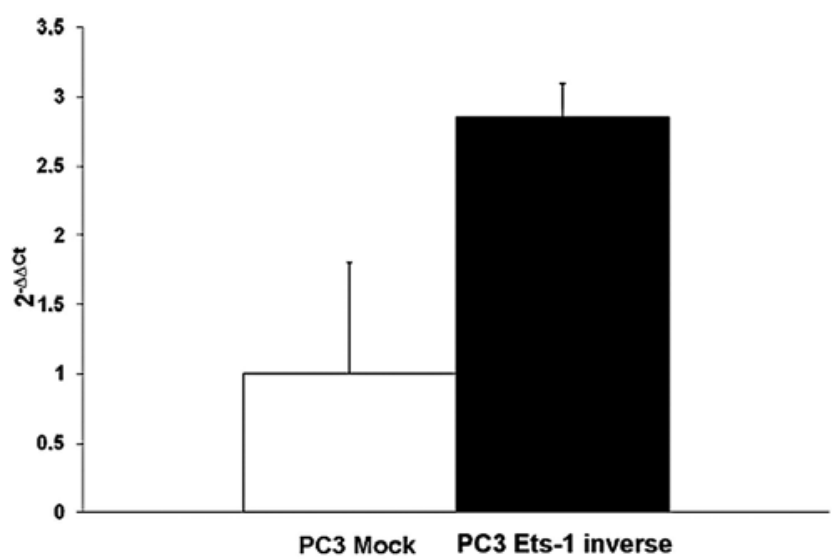

Figure 3. Quantitative reverse transcriptase polymerase chain reaction (qRT-PCR) analysis of FHL2 in PC3 cells. FHL2 mRNA levels in PC3 Mock and PC3 Ets-1 inverse cells determined with qRT-PCR.

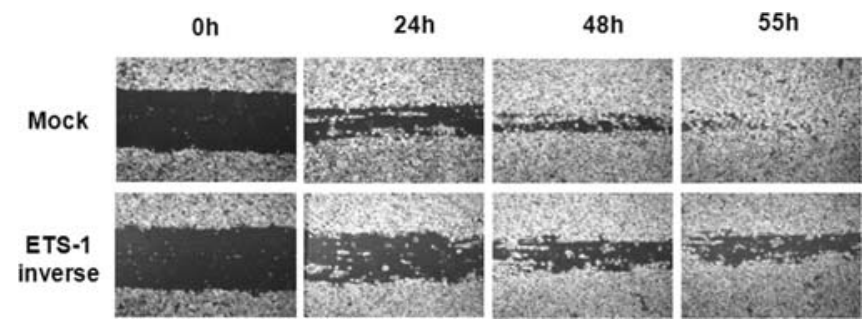

Figure 4. Wound assay: effects of Ets-1 on cell migration. Monolayers of confluent cultures were lightly scratched with a pipette tip and phase contrast images of cultures were taken immediately after wounding $(0 \mathrm{~h})$ and after 24,48 and $55 \mathrm{~h}$.

a 2.36-fold up-regulation of FHL2 in Ets-1 blocked PC3 cells compared to the mock control (Table I). To further verify these findings, we determined the relative expression of FHL2 in the mock control and Ets-1 blocked PC3 cells by qRT-PCR. We found that FHL2 is expressed at a higher level in the Ets-1 blocked cells compared to the mock control cells (Fig. 3).

Ets-1 plays a role in PC3 prostate cancer cell migration. In order to examine the effect of Ets-1 on cell migration we used the wound assay. Wounds were almost completely colonized by PC3 mock control cells in comparison to the PC3 Ets-1 blocked cells after 55 h (Fig. 4). 


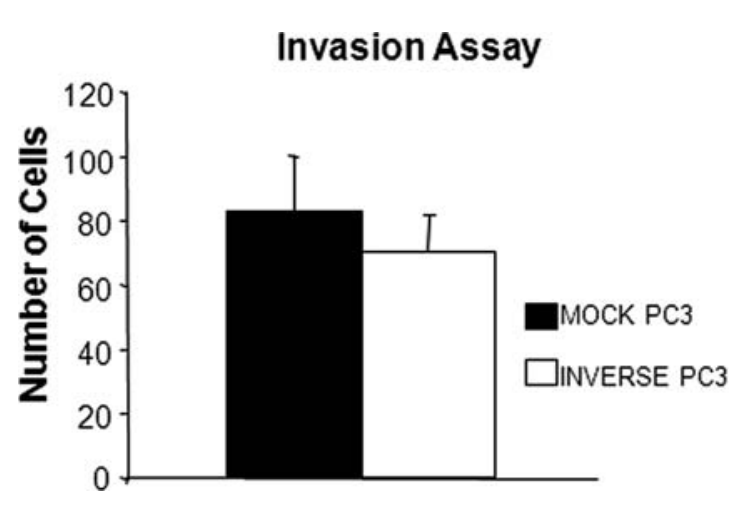

Figure 5. Boyden chamber invasion assays: effect of Ets-1 on cell invasion. Cells were placed into matrigel coated Boyden chambers, and the invading cells were fixed, stained and counted in four different areas after $24 \mathrm{~h}$. Statistical analysis using the T-test revealed no significant differences between mock and inverse PC3 cells.

Ets-1 does not promote invasion of PC3 prostate cancer cells. We investigated the effect of Ets-1 on cell invasion of PC3 prostate cancer cells using the Boyden-chamber assay. The invading cells of the Ets- 1 inverse and mock were counted and the T-test revealed that the difference between Ets-1 inverse and mock is not statistically significant (Fig. 5).

\section{Discussion}

Cancer cell migration is an important step of tumour progression towards metastasis and requires coordinated adhesion and detachment of tumour cells (33). We reported previously that the Ets-1 transcription factor promotes proliferation, migration and invasion in different neoplastic cells such as melanoma, HeLa and glioma cells $(10,11,22)$. In HeLa cells, Ets-1 promotes cell invasiveness and migration, whereas, down-regulation of Ets-1 reduces cell attachment (11).

In the present study, we first investigated whether Ets-1 has a similar effect upon migration and invasion of PC3 prostate cancer cells. Our wound assay showed a decrease in migration in the Ets-1 blocked PC3 cells, indicating a direct role of Ets-1 in this event. However, evaluation of the effect upon invasion was examined using the Boyden chamber, which revealed no significant difference between mock and Ets- 1 blocked cells. These findings suggest that Ets-1 possesses a specificity of action for migration, but not invasion of PC3 prostate cancer cells. Furthermore, it suggests that the role of Ets-1 in migration of PC 3 cells can not be fulfilled by other member of the ETS family which can replace each other under certain conditions (34).

In prostate cancer, the activity of the androgen receptor (AR) is critical for cell proliferation and progression of the disease. Activity of the AR is tightly regulated by different factors and FHL2 (four and a half LIM only protein-2) functions as a nuclear receptor co-regulator that enhances the AR activity and is found in different tissues including the prostate gland $(26,27)$. Additionally, FHL2 is overexpressed and correlated with poor prognosis in prostate cancer $(25,26,28)$. The role of FHL2 in cancer is worth unraveling, as it can function as an oncoprotein or as a tumor suppressor in a tissue-dependent manner, as well as a repressor or an activator of transcription depending on the cell type (35). We therefore, examined whether Ets-1 is implicated in the regulation of FHL2 in PC3 prostate cancer cells. Our results show that FHL2 is up-regulated in the Ets-1 blocked PC3 cells. These findings suggest that Ets-1 is implicated in the regulation of FHL2 and may play a role in a pathway that inhibits the expression of FHL2 in PC3 cells.

In summary, we demonstrate for the first time that Ets-1 is implicated in the regulation of the androgen co-regulator FHL2, and reveals specificity of action for migration, but not invasion of PC3 prostate cancer cells. These findings underline the significance of the role Ets-1 plays in tumor development and suggest that the Ets-1 gene may become a target of novel therapies in the future.

\section{Acknowledgements}

We are grateful for the DFG (Deutsche Forschungsgemeinschaft; German Research Association, grant no. WE 1104/11-1 and the Deutsche Krebshilfe (German Cancer Aid, grant no. 107827) for financial support.

\section{References}

1. Kohn EC: Invasion and metastasis: biology and clinical potential. Pharmacol Ther 52: 235-244, 1991

2. Duffy MJ, McGowan PM and Gallagher WM: Cancer invasion and metastasis: changing views. J Pathol 214: 283-293, 2008.

3. Duffy MJ: Cancer metastasis: biological and clinical aspects. Ir J Med Sci 167: 4-8, 1998.

4. Varga AE, Stourman NV, Zheng Q, et al: Silencing of the Tropomyosin-1 gene by DNA methylation alters tumor suppressor function of TGF-beta. Oncogene 24: 5043-5052, 2005.

5. Bandyopadhyay S, Pai SK, Hirota S, et al: PTEN up-regulates the tumor metastasis suppressor gene Drg-1 in prostate and breast cancer. Cancer Res 64: 7655-7660, 2004.

6. Delgado MD and Leon J: Gene expression regulation and cancer. Clin Transl Oncol 8: 780-787, 2006.

7. Wasylyk B, Hahn SL and Giovane A: The Ets family of transcription factors. Eur J Biochem 211: 7-18, 1993.

8. Oikawa T: ETS transcription factors: possible targets for cancer therapy. Cancer Sci 95: 626-633, 2004.

9. Hashiya N, Jo N, Aoki M, et al: In vivo evidence of angiogenesis induced by transcription factor Ets-1: Ets-1 is located upstream of angiogenesis cascade. Circulation 109: 3035-3041, 2004.

10. Rothhammer T, Hahne JC, Florin A, et al: The Ets-1 transcription factor is involved in the development and invasion of malignant melanoma. Cell Mol Life Sci 61: 118-128, 2004.

11. Hahne JC, Okuducu AF, Kaminski A, Florin A, Soncin F and Wernert N: Ets-1 expression promotes epithelial cell transformation by inducing migration, invasion and anchorageindependent growth. Oncogene 24: 5384-5388, 2005.

12. He J, Pan Y, Hu J, Albarracin C, Wu Y and Dai JL: Profile of Ets gene expression in human breast carcinoma. Cancer Biol Ther 6: 76-82, 2007.

13. Tomlins SA, Rhodes DR, Perner S, et al: Recurrent fusion of TMPRSS2 and ETS transcription factor genes in prostate cancer. Science 310: 644-648, 2005.

14. Furusato B, Tan SH, Young D, et al: ERG oncoprotein expression in prostate cancer: clonal progression of ERG-positive tumor cells and potential for ERG-based stratification. Prostate Cancer Prostatic Dis 13: 228-237, 2010.

15. Behrens P, Rothe M, Wellmann A, Krischler J and Wernert N: The Ets- 1 transcription factor is up-regulated together with MMP 1 and MMP 9 in the stroma of pre-invasive breast cancer. J Pathol 194: 43-50, 2001. 
16. Behrens P, Mathiak M, Mangold E, et al: Stromal expression of invasion-promoting, matrix-degrading proteases MMP-1 and -9 and the Ets 1 transcription factor in HNPCC carcinomas and sporadic colorectal cancers. Int J Cancer 107: 183-188, 2003.

17. Alipov G, Nakayama T, Ito M, et al: Overexpression of Ets-1 proto-oncogene in latent and clinical prostatic carcinomas. Histopathology 46: 202-208, 2005.

18. Hahne JC, Kummer S, Heukamp LC, et al: Regulation of protein tyrosine kinases in tumour cells by the transcription factor Ets-1. Int J Oncol 35: 989-996, 2009.

19. Wernert N, Stanjek A, Kiriakidis S, et al: Inhibition of angiogenesis in vivo by ets-1 antisense oligonucleotides-inhibition of Ets-1 transcription factor expression by the antibiotic fumagillin. Angew Chem Int Ed Engl 38: 3228-3231, 1999.

20. Wernert N, Gilles F, Fafeur V, et al: Stromal expression of c-Ets1 transcription factor correlates with tumor invasion. Cancer Res 54: 5683-5688, 1994.

21. Wernert N, Raes MB, Lassalle P, et al: c-ets1 proto-oncogene is a transcription factor expressed in endothelial cells during tumor vascularization and other forms of angiogenesis in humans. Am J Pathol 140: 119-127, 1992.

22. Sahin A, Vercamer C, Kaminski A, et al: Dominant-negative inhibition of Ets 1 suppresses tumor growth, invasion and migration in rat $\mathrm{C} 6$ glioma cells and reveals differentially expressed Ets 1 target genes. Int J Oncol 34: 377-389, 2009.

23. Heemers HV, Regan KM, Schmidt LJ, Anderson SK, Ballman KV and Tindall DJ: Androgen modulation of coregulator expression in prostate cancer cells. Mol Endocrinol 23: $572-583,2009$

24. Heemers HV, Regan KM, Dehm SM and Tindall DJ: Androgen induction of the androgen receptor coactivator four and a half LIM domain protein-2: evidence for a role for serum response factor in prostate cancer. Cancer Res 67: 10592-10599, 2007.

25. Muller JM, Metzger E, Greschik H, et al: The transcriptiona coactivator FHL2 transmits Rho signals from the cell membrane into the nucleus. EMBO J 21: 736-748, 2002.
26. Kahl P, Gullotti L, Heukamp LC, et al: Androgen receptor coactivators lysine-specific histone demethylase 1 and four and a half LIM domain protein 2 predict risk of prostate cancer recurrence. Cancer Res 66: 11341-11347, 2006.

27. Johannessen M, Moller S, Hansen T, Moens U and Van Ghelue M: The multifunctional roles of the four-and-a-half-LIM only protein FHL2. Cell Mol Life Sci 63: 268-284, 2006.

28. Muller JM, Isele U, Metzger E, et al: FHL2, a novel tissuespecific coactivator of the androgen receptor. EMBO J 19: 359-369, 2000

29. Vancurova I, Miskolci V and Davidson D: NF-kappa B activation in tumor necrosis factor alpha-stimulated neutrophils is mediated by protein kinase Cdelta. Correlation to nuclear Ikappa Balpha. J Biol Chem 276: 19746-19752, 2001.

30. Chen CA and Okayama $\mathrm{H}$ : Calcium phosphate-mediated gene transfer: a highly efficient transfection system for stably transforming cells with plasmid DNA. BioTechniques 6: 632-638, 1988.

31. Kita D, Takino T, Nakada M, Takahashi T, Yamashita J and Sato H: Expression of dominant-negative form of Ets-1 suppresses fibronectin-stimulated cell adhesion and migration through down-regulation of integrin alpha5 expression in U251 glioma cell line. Cancer Res 61: 7985-7991, 2001.

32. Albini A, Benelli R, Noonan DM and Brigati C: The 'chemoinvasion assay': a tool to study tumor and endothelial cell invasion of basement membranes. Int J Dev Biol 48: 563-571, 2004.

33. Yamaguchi $\mathrm{H}$, Wyckoff $\mathrm{J}$ and Condeelis $\mathrm{J}$ : Cell migration in tumors. Curr Opin Cell Biol 17: 559-564, 2005.

34. Laitem C, Leprivier G, Choul-Li S, et al: Ets-1 p27: a novel Ets-1 isoform with dominant-negative effects on the transcriptional properties and the subcellular localization of Ets-1 p51. Oncogene 28: 2087-2099, 2009

35. Kleiber K, Strebhardt K and Martin BT: The biological relevance of FHL2 in tumour cells and its role as a putative cancer target. Anticancer Res 27: 55-61, 2007. 